This item was submitted to Loughborough's Research Repository by the author.

Items in Figshare are protected by copyright, with all rights reserved, unless otherwise indicated.

\title{
A biomechanical evaluation of the combined elevation test
}

PLEASE CITE THE PUBLISHED VERSION

http://dx.doi.org/10.1016/j.ptsp.2016.11.001

\section{PUBLISHER}

(c) Elsevier

VERSION

AM (Accepted Manuscript)

\section{PUBLISHER STATEMENT}

This work is made available according to the conditions of the Creative Commons Attribution-NonCommercialNoDerivatives 4.0 International (CC BY-NC-ND 4.0) licence. Full details of this licence are available at: https://creativecommons.org/licenses/by-nc-nd/4.0/

\section{LICENCE}

CC BY-NC-ND 4.0

\section{REPOSITORY RECORD}

Allen, Samuel J., Gemma C. Phillips, and Steve J. McCaig. 2019. "A Biomechanical Evaluation of the Combined Elevation Test”. figshare. https://hdl.handle.net/2134/23306. 


\title{
A biomechanical evaluation of the combined elevation test
}

\author{
${ }^{1}$ Sam J Allen, ${ }^{2}$ Gemma C Phillips and ${ }^{3}$ Steve J McCaig
}

\begin{abstract}
${ }^{1}$ School of Sport, Exercise and Health Sciences, Loughborough University, Leicestershire, LE11 3TU, UK
${ }^{2}$ Manchester Medical School, University of Manchester, Oxford Road, Manchester, M13 9PT, UK

${ }^{3}$ England and Wales Cricket Board National Cricket Performance Centre, Loughborough University, Leicestershire, LE11 3TU, UK
\end{abstract}

\begin{abstract}
Objectives: To biomechanically evaluate the relationships between the outcome of the Combined Elevation Test, its component joint motions, and thoracic spine angles.

Design: Cross-sectional study.

Setting: Laboratory.

Participants: 18 elite swimmers and triathletes (11 males and 7 females).

Main outcome measures: Combined Elevation Test outcome in forehead and chin positions. Individual joint contributions to test outcome.

Results: No sex differences were found in test components, or between head positions. Test outcome was greater in the forehead position than the chin position $(34.3 \mathrm{~cm}$ vs 30.2 $\mathrm{cm} ; \mathrm{p}<0.001)$. The variables most strongly associated with test outcome were glenohumeral joint flexion $(r=0.86-0.97 ; p<0.001)$, and shoulder retraction $(r=0.75-0.82 ; p<0.001)$. Total thoracic spine angle related strongly to test outcome in females $(r=-0.77--0.88 ; p<0.05)$, but not in males $(r=-0.17--0.24 ; p>0.05)$.

Conclusions: The Combined Elevation Test is an effective screening tool to measure upper limb mobility into shoulder flexion and scapula retraction in both sexes, and thoracic extension in women. It is recommended that the test be performed in the forehead position. If a subject performs poorly on the test, follow up assessments are required to identify the impairment location.
\end{abstract}

Keywords: thoracic spine, shoulder, swimming, triathlon.

\section{INTRODUCTION}

The Combined Elevation Test (CET) is a widely recognised musculoskeletal (MSK) screening tool, used across a variety of sports including, but not limited to: cricket; rugby union; swimming; and triathlon (Blanch, 2004; Gray \& Naylor, 2009; Dennis, Finch, McIntosh, \& Elliot, 2008a; Harvey, 1998). MSK screening is commonly performed to measure potential intrinsic risk factors that may predispose an athlete to injury (DiFiora, 1999); any impairment identified through screening may then be addressed to reduce injury risk. These measurements can be used in prospective studies on uninjured athletes prior to the start of the season and then related to injury outcomes throughout the season (Dennis, Finch, Elliot \& Farhart, 2008). The CET is thought to identify impairments in range of motion during synchronised thoracic extension, glenohumeral joint $(\mathrm{GHJ})$ flexion, and retraction and upward rotation of the scapula (Dennis et al., 2008a; Harvey, 1998). Whilst there are screening tests to assess shoulder flexion (Wilk et al., 2015), scapula upward rotation and retraction (Forthomme, Crielaard, \& Croisier, 2008), and thoracic rotation (Johnson, Kyung-Min, Byung-Kyu, Salibar, \& Grindstaff, 2012), extension of the thoracic spine does not feature in any other field-based assessment tool, thus demonstrating the value of the CET.

When considering the overhead athlete, optimal upper limb and thoracic movement improves force distribution, coordination, and stability; thus reducing compensatory MSK injuries and improving performance outcomes (Blanch, 2004; Dennis et al., 2008a; Gray \& Naylor, 2009; Harvey, 1998; Wilk, Meister, \& Andrews, 2002). In swimming this can relate to the achievement of a streamlined body 
position. In throwing-sports this relates to force generation as well as maximal and sport-specific GHJ range of motion (Culham \& Peat, 1993; Edmondston, Waller, Vallin, Holthe, Noebauer, \& King, 2011; Kebaetse, McClure, \& Pratt, 1999; Kennedy, Visco, \& Press, 2009; Theodorisis \& Ruston, 2002; Wilk et al., 2002).

The format of the CET is broadly consistent in the literature. The subject lays in a prone position on a flat surface, with arms outstretched above the head in approximately 180 degrees of shoulder flexion, elbows locked in full extension and thumbs interlaced. They are subsequently instructed to raise their hands as high as possible whilst maintaining elbow extension. The subject must also keep their head, chest, hips, and legs in contact with the resting surface at all times (Blanch, 2004; Gray \& Naylor, 2009; Dennis et al., 2008a). A notable variation in head position is recognised in both studies conducted by Dennis et al. (2008a; 2008b), where the chin as opposed to the forehead is in contact with the resting surface (Blanch, 2004; Gray \& Naylor, 2009).

The outcome of the CET is recorded in one of two ways. One technique, adopted by Blanch (2004), uses the angle created between the raised humerus and the horizontal; however, the author did not state how this was measured. A technique more widely adopted is measurement, with a tape measure to the nearest $0.1 \mathrm{~cm}$, of the perpendicular height between the resting surface and either the base of the first metacarpal (Dennis et al., 2008a; 2008b; Gray \& Naylor, 2009), or the ulnar styloid (US) (England and Wales Cricket Board [ECB], 2009). The latter method, in conjunction with the subject's forehead resting on the floor, demonstrated results of "almost perfect", both for inter and intra-user reliability (ICC 0.97 and 0.87 ) (Dennis et al., 2008a). Currently no evidence exists for expected normal or sportspecific population values. However, using the first technique, Blanch (2004) states an appropriate outcome for swimmers to be a 5-25 degree humeral angle, while, using the second technique, the ECB employ a value of greater than $20 \mathrm{~cm}$ from the ground to the US (ECB, 2009).

Despite widespread use, there has been no formal evaluation of the CET. Though outcome measures can be reliably recorded, there remains no biomechanical certainty of the absolute and relative movement contributions to test outcome. This is further complicated when considering the impact of variable head position on CET outcomes. The aim of this study was to perform a biomechanical analysis of the CET using a motion-capture system to quantify the geometric contributions of different joint actions to the test outcome; and to assess the relationship between the outcome, and the joint actions and the motion of the thoracic spine. Moreover, the study will investigate the implications of using the two commonly adopted head positions; the two different methods of measurement; and the effect of sex on test outcome and component measures.

\section{METHODS}

Eighteen subjects participated in the study: eleven males (age: $24.5 \mathrm{yrs} \pm 3.1$ yrs, height: $1.82 \mathrm{~m} \pm 0.05 \mathrm{~m}$, weight: $75.3 \mathrm{~kg} \pm 7.4 \mathrm{~kg}$ ); and seven females (age: 23.6 yrs $\pm 4.1 \mathrm{yrs}$, height: $1.73 \mathrm{~m} \pm 0.04 \mathrm{~m}$, weight: $62.8 \mathrm{~kg} \pm 6.8 \mathrm{~kg}$ ). Subjects were either elite swimmers or elite triathletes recruited from English Institute of Sport programmes, who all had previous experience with the CET as part of their regular athletic profiling tests. Study design was approved through the Loughborough University Ethical Advisory Committee and, in accordance with protocol, all subjects provided informed consent to participate and were made aware of their right to withdraw. Strict exclusion criteria were applied during subject recruitment; 
participation was refused if the subject reported any shoulder or back pain within the previous three months, a history of shoulder or spinal surgery, or a self-reported inability to completely extend the elbow joint.

A total of seventeen $14 \mathrm{~mm}$ spherical retroreflective markers were placed in order to represent vertebral and joint centre locations whilst subjects were in the test position (Figure 1). Markers were placed bilaterally around the wrist, elbow, and shoulder joints such that the midpoint of each pair of markers represented the centre of rotation of each joint (Worthington, King, \& Ranson, 2013), and on the spinous processes of T1, T4, T6, T8, and T12 (Figure 1) (Edmonston et al., 2011). All marker locations were palpated and marked out by one of two experienced sports physiotherapists (Masters in Manipulative Therapy and 15 years' experience, last seven full-time in sport), the second investigator (Masters in Sport and Exercise Medicine and 10 years' experience, last five full-time in sport) rechecked each site prior to marker adherence. A 16 camera Vicon (Oxford, UK) motion capture system with Nexus software was used to track the positions of these markers at $100 \mathrm{~Hz}$ throughout each trial.



Figure 1. Retroreflective marker set for automatic motion capture.

The CET was performed in the prone position on the floor, arms outstretched above the head with elbows locked in full extension, fingers interlaced with thumbs pointing towards the ceiling. Subjects were instructed to lift their hands as far off the floor as they could with their elbows extended whilst keeping feet, knees, and hips in contact with the ground at all times. No attempt was made by the assessors to manually ensure full elbow extension, since this would have compromised the ability of the automatic motion capture system to track markers.

Two variations of the test were conducted: 1) forehead resting on the floor; and 2) chin resting on the floor (Figure 2). Two dynamic trials of the CET were completed in each head position. The order in which each head position was tested was randomised between subjects to account for any potential fatigue and learning effects. CET outcome was defined as the maximum wrist joint centre (WJC) height achieved by the dominant hand during the test in each head position for each subject (the WJC height was used instead of US height in order to directly assess the relative contributions of each joint action as described below). 


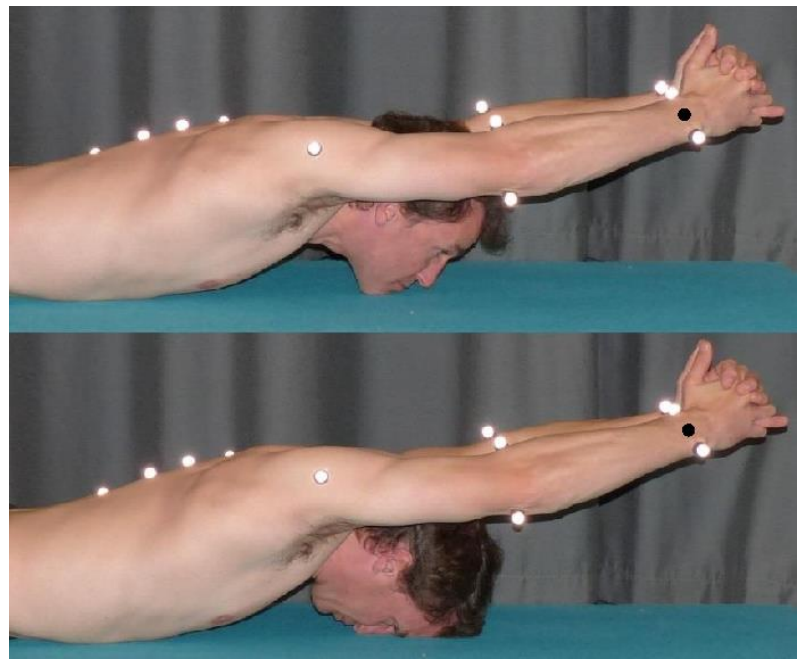

Figure 2. The combined elevation test in the chin (top) and forehead (bottom) positions. Solid black circles indicate WJC position.

Thoracic spine angles were calculated using the methods of Edmondston et al. (2011). Angles were calculated for upper (T1-T4-T6), lower (T4-T8-T12), and total (T1-T6-T12) thoracic regions (Figure 3).

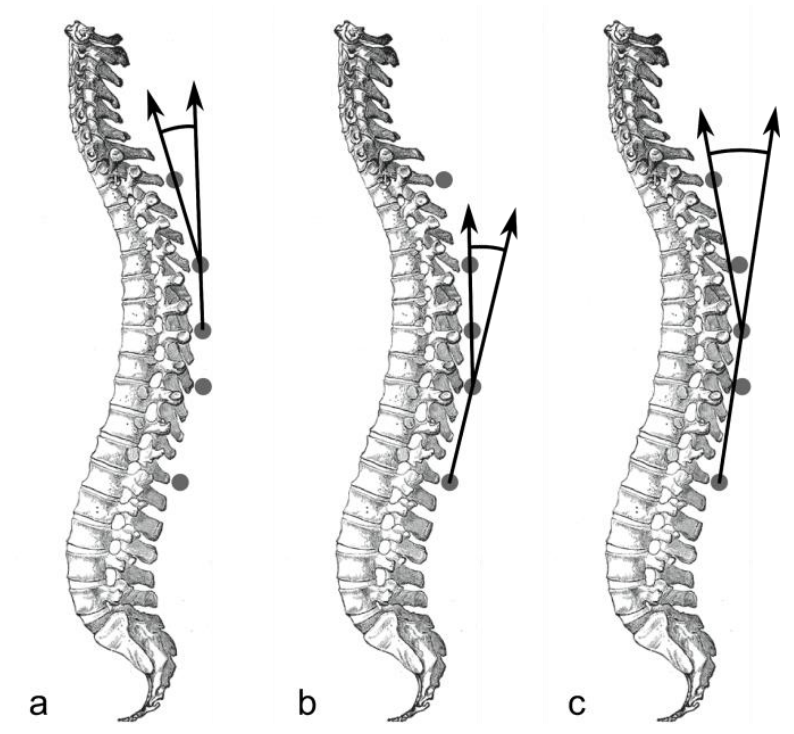

Figure 3. Upper (a), lower (b), and total (c) thoracic spine angles calculated from skin-mounted markers.

Geometry of the test was determined by assessing the contribution of the following to test outcome in the sagittal plane (Figure 4):

1) Shoulder joint centre (SJC) height - the vertical position of SJC at the point of maximal WJC height. This measurement was used as a proxy for retraction and upward rotation of the scapula, since this is impossible to measure accurately with skin-mounted marker systems due to soft tissue artefact (Matsui, Shimada, \& Andrew, 2006).

2) Shoulder flexion - the theoretical contribution of the GHJ flexion angle to WJC height calculated using the GHJ flexion angle relative to the horizontal at the 
point of maximal WJC height, and segment lengths obtained from joint centre coordinates.

3) Elbow flexion - the theoretical contribution of elbow joint flexion to WJC height calculated from elbow flexion angle and segment lengths obtained from joint centre coordinates.



Figure 4. The contributions of each joint to test performance: hsj is the height of the shoulder joint centre; hsf is the contribution of shoulder flexion, assuming no elbow flexion; hef is the contribution of elbow flexion; and htot is the total vertical displacement of the WJC. (n.b. hsf and hef values can be negative).

All of the above measures were absolute values taken at the point of maximum WJC height. This was preferred to calculating displacements from some arbitrary 'rest' position, since this was not considered to be representative of the genuine contribution of these joint motions to test outcome.

To produce the second measurement of the CET outcome in degrees, as used by Blanch (2004), the angle created between the elbow joint centre (EJC) and SJC, representative of the humerus, and the horizontal, was recorded at the point of maximal WJC height.

It was established that all data were normally distributed using a one-sample Kolmogorov-Smirnov test, indicating the use of parametric statistics was acceptable. Differences between sexes were evaluated using independent samples Student's ttests, and between test position using paired-samples Student's t-tests. Similarities were evaluated using Pearson's linear correlations and simple linear regression. All data analysis was performed using MATLAB® (R2013b, Mathworks, Natick, MA, USA).

\section{RESULTS}

All displacement data is presented in centimetres, and angle data in degrees, since these are the units most commonly used in practice. The effects of the decision to use WJC instead of US displacement as the test outcome was assessed by correlating the two across all trials $(n=36)$ and it was found that they were extremely closely related $(r=0.99)$, so this was considered to be acceptable. Linear regression indicated that the two measures can be equated using the following relationship:

$$
U=1.0547 W-4.557 \text {, }
$$

where $U$ is the US displacement, and $W$ is the WJC displacement, both in centimetres.

CET results were compared between males $(n=11)$ and females $(n=7)$ in both head positions for: test outcome; individual joint contributions; and thoracic spine angles, and no differences were found ( $>0.05)$ (Table 1), therefore all further analyses considered males and females as one group $(n=18)$. Figure 5 shows test 
outcome and contributions of individual components in each test position for all subjects.

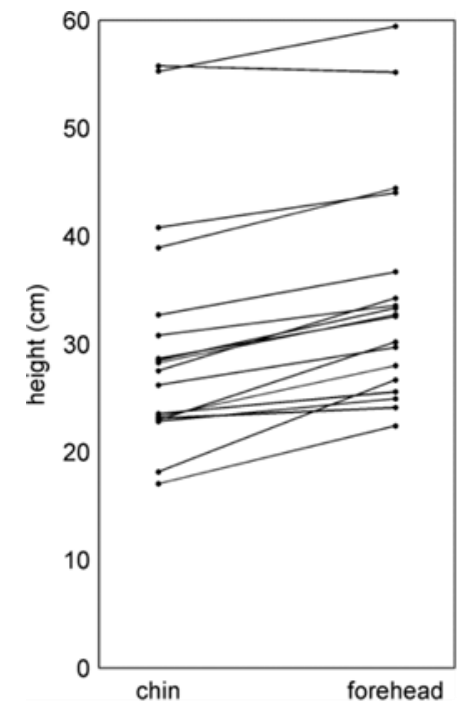

a)

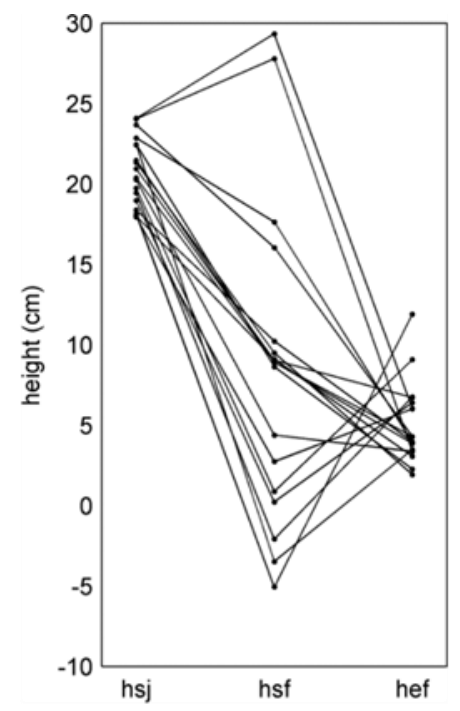

b)

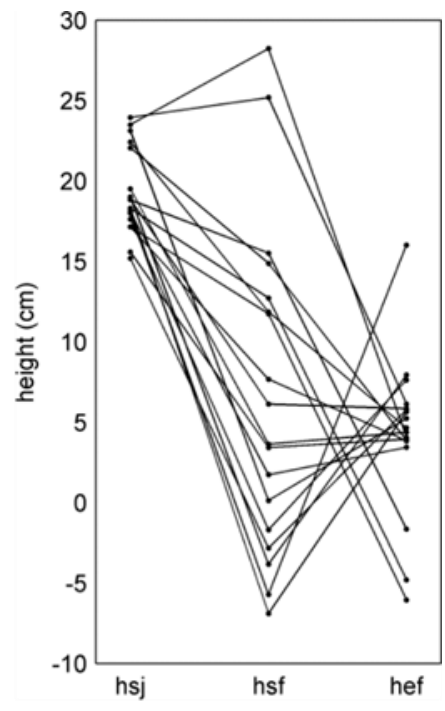

c)

Figure 5. Dot plots showing a) CET performance in the two head positions; b) Contributions to performance from different joint actions in the forehead position; c) Contributions to performance from different joint actions in the chin position.

Table 1. Test measures (mean $\pm \mathrm{SD}$ ) between positions and sexes.

\begin{tabular}{crrrr}
\hline & \multicolumn{2}{c}{ forehead } & \multicolumn{1}{c}{ chin } \\
& \multicolumn{1}{c}{ male } & \multicolumn{1}{c}{ female } & \multicolumn{1}{c}{ male } & \multicolumn{1}{c}{ female } \\
\hline $\mathbf{h}_{\text {tot }}(\mathbf{c m})$ & $34.0 \pm 10.0$ & $34.8 \pm 11.7$ & $30.5 \pm 10.9$ & $29.8 \pm 12.2$ \\
$\mathbf{h}_{\mathbf{s j}}(\mathbf{c m})$ & $21.3 \pm 2.0$ & $20.0 \pm 2.3$ & $19.8 \pm 2.5$ & $18.5 \pm 3.0$ \\
$\mathbf{h}_{\mathbf{s f}}(\mathbf{c m})$ & $7.8 \pm 9.9$ & $9.6 \pm 9.8$ & $6.6 \pm 10.5$ & $7.0 \pm 10.3$ \\
$\mathbf{h}_{\text {ef }}(\mathbf{c m})$ & $4.9 \pm 3.0$ & $5.2 \pm 1.5$ & $4.1 \pm 5.9$ & $4.3 \pm 3.0$ \\
$\mathbf{T}_{\mathbf{1}}-\mathbf{T}_{\mathbf{1 2}}\left(^{\circ}\right)$ & $12.2 \pm 5.3$ & $13.3 \pm 3.7$ & $12.6 \pm 4.9$ & $12.8 \pm 4.4$ \\
$\mathbf{T}_{\mathbf{1}}-\mathbf{T}_{\mathbf{8}}\left(^{\circ}\right)$ & $10.2 \pm 4.9$ & $8.9 \pm 5.4$ & $9.9 \pm 4.7$ & $8.6 \pm 4.2$ \\
$\mathbf{T}_{\mathbf{4}}-\mathbf{T}_{\mathbf{1 2}}\left(^{\circ}\right)$ & $10.5 \pm 3.6$ & $11.7 \pm 2.5$ & $11.1 \pm 3.3$ & $12.0 \pm 3.2$ \\
\hline
\end{tabular}

Where hsj is the height of the shoulder joint centre; hsf is the contribution of shoulder flexion; hef is the contribution of elbow flexion; htot is the test outcome; T1-T12 is the total thoracic spine angle; T1-T8 is the upper thoracic spine angle; and T4-T12 is the lower thoracic spine angle.

When comparing the test outcome and components in the two head positions, differences were found (Table 2): htot in the forehead position $(34.3 \mathrm{~cm} \pm 10.3 \mathrm{~cm})$ was significantly greater than the chin position $(30.2 \mathrm{~cm} \pm 11.1 \mathrm{~cm})(p<0.001)$; of the contributions of the joint positions, hsj was consistently greater in the forehead position $(20.8 \mathrm{~cm} \pm 2.1 \mathrm{~cm})$ than the chin position $(19.3 \mathrm{~cm} \pm 2.7 \mathrm{~cm})(p<0.001)$ however there were no significant differences in hsf or hef. Of the thoracic spine angles the only significant difference was in the lower spine angle T4-T12 which was smaller in the forehead position $\left(11.0^{\circ} \pm 3.2^{\circ}\right)$ than the chin position $\left(11.4^{\circ} \pm 3.2^{\circ}\right)$ $(p=0.02)$. 
Table 2. Test measures (mean \pm SD) in both head positions with associated p-values, $95 \%$ confidence intervals $(\mathrm{Cl})$ and effect sizes (Cohen's d) for differences between positions.

\begin{tabular}{lrrrrr}
\hline measure & \multicolumn{1}{c}{$\begin{array}{c}\text { forehead } \\
(\mathbf{n}=\mathbf{1 8})\end{array}$} & \multicolumn{1}{c}{$\begin{array}{c}\text { chin } \\
(\mathbf{n}=\mathbf{1 8})\end{array}$} & $\mathbf{p}$-value & $\mathbf{9 5 \%} \mathbf{~ C l}$ & Cohen's d \\
\hline $\mathbf{h}_{\text {tot }}(\mathbf{c m})$ & $34.3 \pm 10.3$ & $30.2 \pm 11.1$ & $<0.001$ & $-5.19,-2.98$ & 2.75 \\
$\mathbf{h}_{\mathbf{s j}}(\mathbf{c m})$ & $20.8 \pm 2.1$ & $19.3 \pm 2.7$ & $<0.001$ & $-2.14,-0.96$ & 1.97 \\
$\mathbf{h}_{\mathbf{s f}}(\mathbf{c m})$ & $8.5 \pm 9.6$ & $6.8 \pm 10.1$ & 0.33 & $-5.32,1.91$ & 0.33 \\
$\mathbf{h}_{\text {ef }}(\mathbf{c m})$ & $5.0 \pm 2.5$ & $4.2 \pm 4.8$ & 0.62 & $-4.22,2.56$ & 0.16 \\
$\mathbf{T}_{\mathbf{1}}-\mathbf{T}_{\mathbf{1 2}}\left({ }^{\circ}\right)$ & $12.6 \pm 4.6$ & $12.7 \pm 4.6$ & 0.95 & $-0.59,0.62$ & 0.12 \\
$\mathbf{T}_{\mathbf{1}}-\mathbf{T}_{\mathbf{8}}\left({ }^{\circ}\right)$ & $9.7 \pm 5.0$ & $9.4 \pm 4.4$ & 0.56 & $-1.29,0.72$ & 0.22 \\
$\mathbf{T}_{\mathbf{4}}-\mathbf{T}_{\mathbf{1 2}}\left({ }^{\circ}\right)$ & $11.0 \pm 3.2$ & $11.4 \pm 3.2$ & 0.02 & $0.10,0.83$ & 0.76 \\
\hline
\end{tabular}

Where hsj is the height of the shoulder joint centre; hsf is the contribution of shoulder flexion; hef is the contribution of elbow flexion; htot is the test outcome; T1-T12 is the total thoracic spine angle; T1-T8 is the upper thoracic spine angle; and T4-T12 is the lower thoracic spine angle.

In an attempt to assess the relationships between the different test components and and test performance, hsj, hsf, hef, and the three thoracic spine angles were correlated to htot in both test positions at the point of maximum WJC displacement (Table 3). The variables with the strongest relationship to test performance were hsj and hsf which were strongly correlated to CET outcome in both test positions, but hef had minimal influence. In each test position total thoracic spine angle correlated negatively with test performance more strongly than either upper or lower thoracic spine angle, indicating that a smaller (more extended) T1-T12 angle was related to an increased htot, but none was statistically significantly related (Table 3 ). The three most strongly correlated variables hsj, hsf, and T1-T12 were then regressed against htot in order to demonstrate the predictive relationships between the components and the test outcome (Figure 6). In order to determine whether sex differences existed in these relationships, hsj, hsf, and T1-T12 were also regressed against htot for males and females independently in both head positions (Table 4 and Figure 7). These regressions indicated that, whilst strong statistically significant relationships persisted for both sexes between hsj and hsf, and htot in both head positions ( $\mathrm{r} 2$ > 0.5), T1-T12 angle predicted a large and statistically significant amount of the variance in htot in both head positions in females ( $r 2=0.60$ and 0.78 ) but almost none in males ( $r 2=0.06$ and 0.03 ) (Table 4 and Figure 7). 
Table 3. Results of correlations between individual test components and test outcome with associated p-values and $95 \%$ confidence intervals $(\mathrm{Cl})$ for $r$ values.

\begin{tabular}{lcccccc}
\hline & \multicolumn{3}{c}{$\begin{array}{c}\text { forehead } \\
(\mathbf{n = 1 8})\end{array}$} & \multicolumn{3}{c}{$\begin{array}{c}\text { chin } \\
(\mathbf{n = 1 8})\end{array}$} \\
measure & $\mathrm{r}$ & $\mathrm{p}$ & $95 \% \mathrm{Cl}$ & $\mathrm{r}$ & \multicolumn{1}{c}{$\mathrm{p}$} & $95 \% \mathrm{Cl}$ \\
\hline $\mathbf{h}_{\mathbf{s j}}$ & 0.75 & $<0.001$ & $0.43,0.90$ & 0.82 & $<0.001$ & $0.57,0.93$ \\
$\mathbf{h}_{\mathbf{s f}}$ & 0.97 & $<0.001$ & $0.92,0.99$ & 0.86 & $<0.001$ & $0.65,0.95$ \\
$\mathbf{h}_{\mathbf{e f}}$ & -0.22 & 0.38 & $-0.62,0.27$ & 0.04 & 0.87 & $-0.43,0.50$ \\
$\mathbf{T}_{\mathbf{1}}-\mathbf{T}_{\mathbf{1 2}}$ & -0.40 & 0.10 & $-0.73,0.08$ & -0.44 & 0.07 & $-0.75,0.03$ \\
$\mathbf{T}_{\mathbf{1}}-\mathbf{T}_{\mathbf{8}}$ & -0.09 & 0.71 & $-0.54,0.39$ & -0.18 & 0.46 & $-0.60,0.31$ \\
$\mathbf{T}_{\mathbf{4}}-\mathbf{T}_{\mathbf{1 2}}$ & -0.24 & 0.33 & $-0.64,0.26$ & -0.34 & 0.17 & $-0.70,0.15$ \\
\hline
\end{tabular}

Where hsj is the height of the shoulder joint centre; hsf is the contribution of shoulder flexion; hef is the contribution of elbow flexion; T1-T12 is the total thoracic spine angle; T1-T8 is the upper thoracic spine angle; and T4-T12 is the lower thoracic spine angle.

Table 4. Results of regressions between individual test components and test outcome with associated p-values.

\begin{tabular}{|c|c|c|c|c|c|c|c|c|}
\hline \multirow{3}{*}{ measure } & \multicolumn{4}{|c|}{ forehead } & \multicolumn{4}{|c|}{ chin } \\
\hline & \multicolumn{2}{|c|}{ male } & \multicolumn{2}{|c|}{ female } & \multicolumn{2}{|c|}{ male } & \multicolumn{2}{|c|}{ female } \\
\hline & $r^{2}$ & $p$ & $r^{2}$ & $p$ & $r^{2}$ & $p$ & $r^{2}$ & $p$ \\
\hline $\mathbf{h}_{\mathbf{s j}}$ & 0.56 & 0.004 & 0.74 & 0.006 & 0.62 & 0.008 & 0.81 & 0.013 \\
\hline $\mathbf{h}_{\mathbf{s f}}$ & 0.95 & 0.002 & 0.95 & 0.002 & 0.66 & $<0.001$ & 0.87 & $<0.001$ \\
\hline$T_{1}-T_{12}$ & 0.06 & 0.61 & 0.60 & 0.009 & 0.03 & 0.46 & 0.78 & 0.04 \\
\hline
\end{tabular}

Where hsj is the height of the shoulder joint centre; hsf is the contribution of shoulder flexion; and T1-T12 is the total thoracic spine angle.
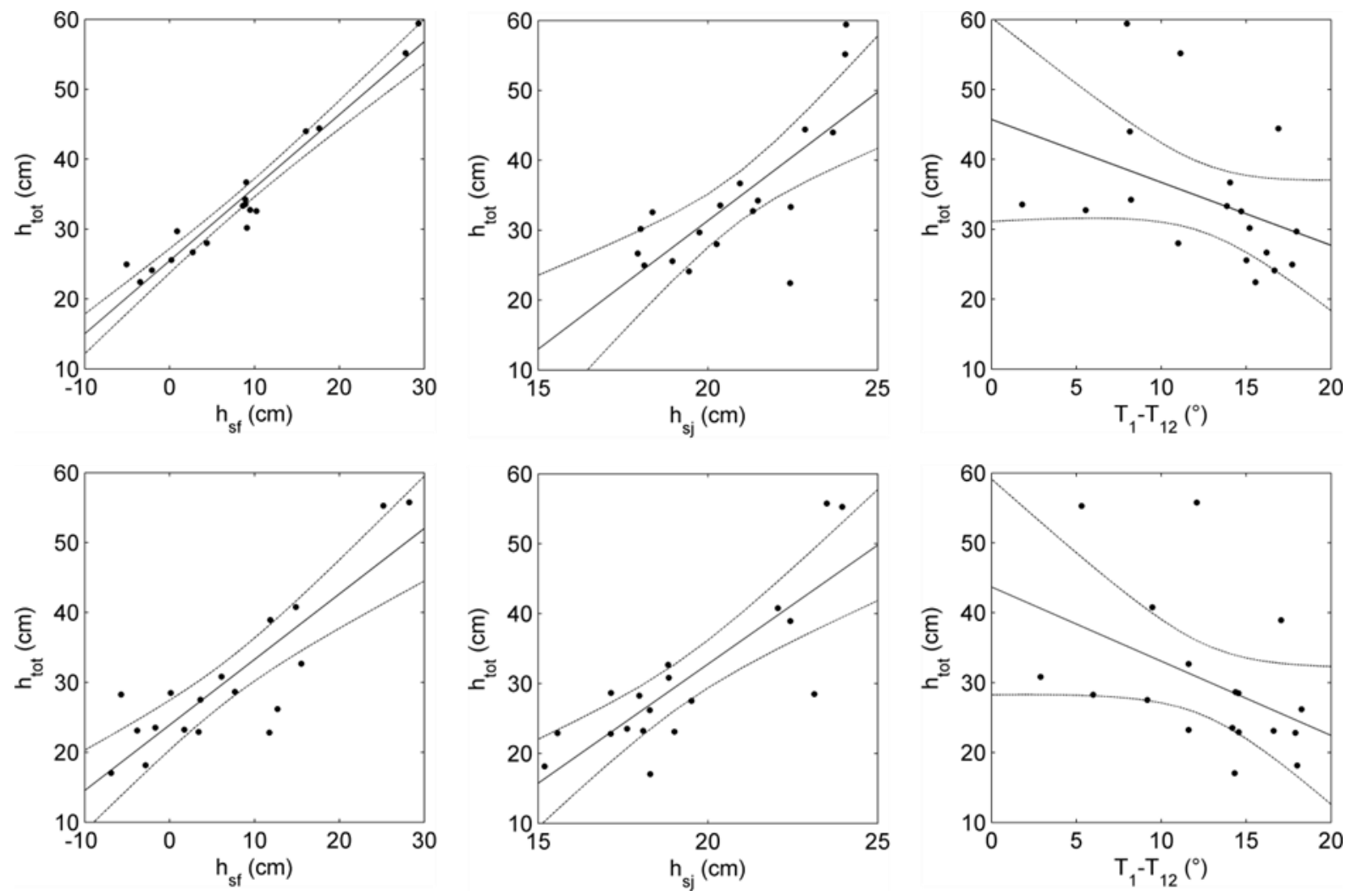

Figure 6. The contributions of each joint to test performance: hsj is the height of the shoulder joint centre; hsf is the contribution of shoulder flexion, assuming no elbow flexion; hef is the contribution of elbow flexion; and htot is the total vertical displacement of the WJC. (n.b. hsf and hef values can be negative. 

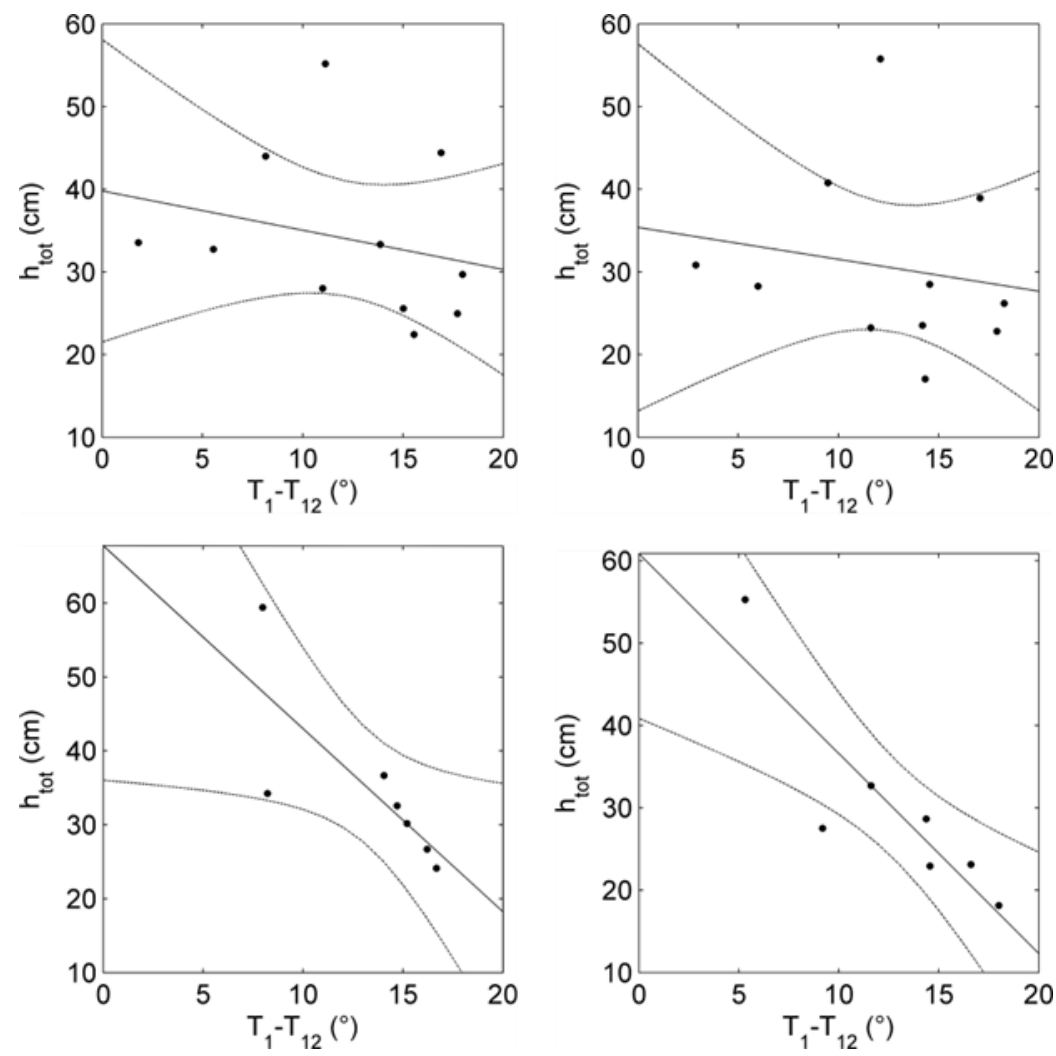

Figure 7. Dot plots with least squares regression lines (Broken lines indicate 95\% confidence intervals) showing the relationships between test outcome (htot) and total thoracic spine angle (T1T12) in the forehead (left) and chin (right) positions in males (top row), and females (bottom row).

Lastly, comparing two different methods of measuring the outcome of the test, htot versus humeral angle, for all trials $(n=36)$ indicated that the two were strongly, but not perfectly, related $(r=0.93, p<0.001)$.

\section{DISCUSSION}

This is the first study to have analysed the biomechanics of the CET and described the joint contributions to the test outcome, as well as to report normative values for any population. It has been proposed that the CET measures combined thoracic extension, shoulder girdle flexion, and scapula retraction (Harvey, 1998), but no previous study has investigated how these components relate to test outcome. This study demonstrated that movement occurred in each of these regions during the test, and quantified their relative contributions to test outcome. This indicates that the CET is useful as a screening tool for upper limb mobility.

In this population of elite swimmers and triathletes the mean outcome of the CET was $34.3 \mathrm{~cm}( \pm 10.3 \mathrm{~cm})$ in the forehead position and $30.2 \mathrm{~cm}( \pm 11.1 \mathrm{~cm})$ in the chin position. When males and females were compared no differences were found between the two groups, neither in test outcome, nor in the individual components of the test (Table 1). This indicates that for elite swimmers and triathletes it would seem reasonable to suggest that males and females could be assessed using the same population norms. As no other studies have published normative values it is difficult to compare these results to other populations. Both Blanch (2004) and the ECB (ECB, 2009) reported appropriate outcomes for the CET for swimmers and cricketers $\left(5^{\circ}-25^{\circ}\right.$ humeral angle, and $>20 \mathrm{~cm}$ US height respectively), however neither reported any normative data for their populations, nor a rationale for the proposed outcomes. Only approximately $45 \%$ of the participants in this study were within 
Blanch's (2004) range for swimmers, and the majority of those outside the range, a further $45 \%$, were below $5^{\circ}$.

As all of the subjects in this study were uninjured, the effect of pain and injury on the performance of the CET could not be assessed; and since we did not follow subjects over a period of time we could not establish how performance on the CET relates to injury risk. Further studies should report normative outcomes across a variety of populations and investigate the effect of pain and injury on performance of the test, and prospective studies should investigate the relationships between the outcome of the CET and injury risk and/or sporting performance.

The outcome of the test was shown to be significantly different between the two head positions (Table 2; Figure 5), when the test was carried out with the forehead in contact with the ground subjects were able to raise their WJC significantly higher than when the chin was in contact with the ground. In terms of the test components, only the height of the shoulder joint, which was used as a proxy for scapular retraction, was significantly different between head positions, and this difference represented a large effect size (Cohen, 1988). Subjects were able to retract their shoulder joints significantly further in the forehead position than in the chin position, indicating that this retraction was limited by the extension of the cervical spine brought about by maintaining contact between the chin and the ground. As some of the muscles that retract the scapula (upper trapezius, middle trapezius, levator scapulae, rhomboids) attach to the cervico-thoracic spine, changes in cervical posture may alter the position on the length-tension curve of these muscle. Consequently, whilst performing the test in the chin position these muscles may be operating at a length that is shorter than optimal which could compromise their ability to produce force in order to retract the scapula. Performing the test in the chin position was also associated with increased variability in the test outcome and all three joint components of the test, when compared with the forehead position; this was especially apparent in elbow flexion where the standard deviation almost doubled (Table 2; Figure 5). It is possible, since subjects did not perform as well in the test in the chin position as in the forehead position, that they compensated for this by flexing their elbows. Since no attempt was made to restrict the elbow motion of the subjects a number did exhibit some elbow flexion (Table 2; Figure 5). The range of this elbow flexion was more pronounced in the chin position than the forehead position, however across all subjects, elbow flexion was not related to test outcome in either head position (Table 3). So across a population of subjects who have simply been instructed to maintain full elbow extension, practitioners can be confident that the test outcome is little influenced by elbow flexion. However, as it is possible for an individual to substantially improve his or her test outcome by flexing at the elbow, steps should be taken to minimise this if possible; this can be achieved by having one assessor monitoring elbow flexion whilst another records the test outcome. Relationships between test components and outcome were similar between head positions, and therefore we recommend that the CET be performed in the forehead position because this resulted in more consistent results and less elbow flexion.

Of the thoracic spine angles, only the lower spine angle T4-T12 was significantly different between test positions (Table 2); however, the difference was practically so small that caution should be exercised when attributing clinical significance to this result.

The results of the correlations between test components and outcomes indicated that the component with the strongest relationship to test outcome was 
GHJ flexion; regressions indicated that variance in this component predicted $94 \%$ of the variance in test performance in the forehead position, and $74 \%$ in the chin position - these values can be obtained by squaring the $r$ values in Table 3 (Figure 6). This indicates that performance in the CET is very strongly related to range of GHJ flexion. The second strongest predictor of test outcome was shoulder joint retraction; variance in this component accounted for $56 \%$ and $67 \%$ of the variance in test outcome in the forehead and chin position respectively (Table 3; Figure 6). These relationships persisted when regressions were considered at an individual sex level, although they were typically stronger in females than males (Table 4, Figure 7). The fact that the combined percentages of variance explained by these two variables totals much more than $100 \%$ in all analyses indicates that they are also related to each other; that is the ability to retract the shoulder is related to the ability to flex the GHJ. This makes sense given the results of McClure et al. (2001) who found that glenohumeral flexion in the coronal plane was accompanied by scapular upward rotation, posterior tilting, and external rotation.

Variance in total thoracic spine angle predicted $16 \%$ and $19 \%$ of the variance in test outcome in the forehead and chin position respectively across the whole group (Table 3; Figure 6). The $r$ values ( -0.4 and -0.44 respectively) can still be considered to represent a medium effect size (Cohen, 1992) however this was not statistically significant in either case $(p=0.07$ and $p=0.1)$. When considered at an individual sex level different trends appeared; women showed a strong relationship between thoracic spine angle and test outcome, and men showed practically no relationship at all (Table 4, Figure 7). The CET is reported to be an indicator of thoracic mobility and, given these results, it can be concluded that this is likely to be related to test outcome in women within the test population, only slightly more weakly than GHJ flexion and shoulder retraction. However, within this test population it does not seem that CET outcome relates to thoracic spine angle in men. The fact that strong and significant relationships were found despite the small sample of female athletes is an indicator that this result is likely to be meaningful. Studies on larger samples of athletes would be necessary to see whether a relationship also exists in males that was masked in this study by low statistical power, but there were no indications of this in our data. There is no obvious anatomical explanation of why this difference exists between males and females, especially as there were no differences between these groups in thoracic extension during the test.

When comparing different outcome measures of the test, humeral angle was strongly related to wrist joint centre height, with a shared variance of $86 \%$. This however does leave a proportion of the variance unexplained. Given that it has been shown that retraction of the shoulder joint is an important component of the test outcome, and that thoracic spine motion may also be related, it would seem sensible to use wrist height if the test is being used as an indicator of mobility in these joints. If the only concern is $\mathrm{GHJ}$ flexion range then humeral angle is likely to be a suitable measure.

The CET does appear to be a useful screening tool to identify insufficient movement into upper limb elevation, but further studies are required to identify ideal outcome ranges for various population groups. However, the CET does not indicate which joint motions contribute to the outcome, and if a subject had a poor outcome on the CET, follow up tests would be necessary to identify where the impairment was located e.g. passive shoulder flexion (Wilk et al., 2015), pectoralis minor length (Borstad, 2008), and thoracic intervertebral joint motion (Brismée et al., 2006). This would help guide the practitioner towards an appropriate intervention, e.g. specific stretching of pectoralis minor if restricted mobility were apparent, to improve 
performance on the test for that subject. Future studies could investigate the effects of specific treatment techniques and exercises on performance in the CET.

Using skin-mounted markers to infer the motion of underlying joints will inevitably have introduced some error into the data in this study. However, the CET does not involve an impact or substantial soft tissue movement likely to introduce artefacts, and the data analysis did not require differentiation of position data which greatly amplifies these artefacts. The marker positions were consistently checked by two investigators and the strong relationships observed between the test outcome and the joint level data indicates that they are likely to be a reliable indicator of joint motions. Despite representing the total number of elite swimmers and triathletes available to partake in the study, samples sizes of males and females were not large, therefore it is possible that differences between sexes could have been masked by low statistical power. However, if these differences exist they are likely to be small since none remotely approached significance in the current analysis.

\section{CONCLUSION}

The results indicate that the CET is an effective screening tool to measure upper limb mobility into shoulder flexion and scapula retraction in males and females, and thoracic extension in females. It is recommended that the test be performed in the forehead position as this produced more consistent results and reduced the amount of elbow flexion. When considered across a population of elite swimmers and triathletes there were no differences between males and females in CET outcome or individual test components, this suggests that normative data may be applied to both males and females in this population. As a screening tool the CET provides a quick measure of upper limb mobility, however if a subject performs poorly on the CET, follow up tests are required to identify where the impairment is located and design a suitable treatment intervention.

\section{ACKNOWLEDGEMENTS}

The authors would like to acknowledge the support of the English Institute of Sport in allowing their athletes to participate in this study.

\section{REFERENCES}

Blanch P. Conservative Management of Shoulder Pain in Swimming. Phys Ther in Sport. 2004;5:109-124. http://dx.doi.org/10.1016/j.ptsp.2004.05.002

Borstad JD. Measurement of Pectoralis Minor Muscle Length: Validation and Clinical Application. J Orthop Sports Phys Ther. 2008;38:169-174. http://dx.doi.org/10.2519/jospt.2008.2723

Brismée JM, Gipson D, Ivie D, Lopez A, Moore M, Matthijs O, Phelps V, Sawyer S, Sizer P. Interrater Reliability of a Passive Physiological Intervertebral Motion Test in the Mid-Thoracic Spine. J Manip Physiol Therap. 2006;29:368-373. http://dx.doi.org/10.1016/j.jmpt.2006.04.009

Cohen J. Statistical power analysis for the behavioral sciences (2nd ed.). 1988; Hillsdale, NJ: Lawrence Earlbaum Associates.

Cohen J. A power primer. Psych Bull. 1992;112:155-159. http://dx.doi.org/10.1037/0033-2909.112.1.155

Culham P, Peat M. Functional Anatomy of the Shoulder Complex. J Orthop Sports Phys Ther. 1993;18:342-350. http://dx.doi.org/10.2519/jospt.1993.18.1.342 
Dennis RJ, Finch CF, Elliot BC, Farhart PJ. The reliability of musculoskeletal screening tests used in cricket. Phys Ther in Sport. 2008a;9:25-33. http://dx.doi.org/10.1016/j.ptsp.2007.09.004

Dennis RJ, Finch CF, McIntosh AS, Elliott BC. Use of Field-Based Tests to Identify Risk Factors for Injury to Fast Bowlers in Cricket. Br J Sports Med. 2008b;42:477482. http://dx.doi.org/10.1136/bjsm.2008.046698

DiFiora, J. Overuse injuries in children and adolescents. Phys and Sports Med 1999; 75-89. http://dx.doi.org/10.3810/psm.1999.01.652

Edmondston SJ, Waller R, Vallin P, Holthe A, Noebauer A, King E. Thoracic Spine Extension Mobility in Young Adults: Influence of Subject Position and Spinal Curvature. J Orthop Sports Phys Ther. 2011;241:266-273. http://dx.doi.org/10.2519/jospt.2011.3456

England and Wales Cricket Board (ECB) Science and Medicine Department. ECB Screening Protocols. 2009. Unpublished.

Forthomme, B., Crielaard, J. \& Croisier, J. Scapular positioning in athlete's shoulder : particularities, clinical measurements and implications. Sports Med. 2008; 38:369386. http://dx.doi.org/10.2165/00007256-200838050-00002

Gray J, Naylor R. Musculoskeletal assessment form, BokSmart Winners plays smart, South African Rugby Union. 2009;19-20.

Harvey D. Screening test protocols. Pre-participation screening of athletes: A Program developed by the Australia Institute of Sport, Olympic Athlete Program and the Australian Sports Injury Prevention Program. Australian Sports Commission. Taskforce: Canberra, Australia. 1998.

Johnson KD, Kyung-Min K, Byung-Kyu Y, Salibar SA, Grindstaff TL. Reliability of thoracic spine rotation range-of-motion in healthy adults. J Athl Train. 2012;47:5260.

Kebaetse M, McClure P, Pratt NA. Thoracic Position Effect on Shoulder Range of Motion, Strength, and Three-Dimensional Scapular Kinematics. Arch Phys Med Rehab. 1999;80:945-950. http://dx.doi.org/10.1016/S0003-9993(99)90088-6

Kennedy DJ, Visco CJ, Press J. Current Concepts for Shoulder Training in the Overhead Athlete. Curr Sports Med Reports. 2009;8:154-162. http://dx.doi.org/10.1249/JSR.0b013e3181a64607

Matsui K, Shimada K, Andrew PD. Deviation of skin marker from bone target during movement of the scapula. J Orthop Sci. 2006;11:180-184. http://dx.doi.org/10.1007/s00776-005-1000-y

McClure PW, Michener LA, Sennett BJ, Karduna AR. Direct 3-Dimensional Measurement of Scapular Kinematics During Dynamic Movements in Vivo. J of Shoulder Elbow 2001;10:269-277. http://dx.doi.org/10.1067/mse.2001.112954

Theodorisis D., Ruston S. The Effect of Shoulder Movements on Thoracic Spine 3D Motion. Clin Biomech. 2002;17:418-421. http://dx.doi.org/10.1016/S02680033(02)00026-8

Wilk KE, Meister K, Andrews JR. Current Concepts in the Rehabilitation of the Overhead Throwing Athlete. Am J Sports Med. 2002;30:136-151.

Wilk KE, Macrina LC, Fleisig GS, Aune KT, Porterfield RA, Harker P, Evans TJ, Andrews JR. Deficits in Glenohumeral Passive Range of Motion Increase Risk of Shoulder Injury in Professional Baseball Pitchers. A Prospective Study. Am J Sports Med. 2015;43:2379-2385. http://dx.doi.org/10.1177/0363546515594380 
Worthington PJ, King MA, Ranson CA. Relationships between fast bowling technique and ball release speed in cricket, J Appl Biomech. 2013;29:78-84. 